\title{
Effect of Mix Proportions on Rheological and Hardened Properties of Composite Cement Pastes
}

\author{
Mohammed Sonebi* and Denis McKendry
}

School of Planning, Architecture and Civil Engineering, Queen's University Belfast, Belfast, Northern Ireland, UK

\begin{abstract}
There is an increasing need to identify the effect of mix composition on the rheological properties of composite cement pastes using simple tests to determine the fluidity, the cohesion and other mechanical properties of grouting applications such as compressive strength. This paper reviews statistical models developed using a fractional factorial design which was carried out to model the influence of key parameters on properties affecting the performance of composite cement paste. Such responses of fluidity included mini-slump, flow time using Marsh cone and cohesion measured by Lombardi plate meter and unit weight, and compressive strength at $3 \mathrm{~d}, 7 \mathrm{~d}$ and $28 \mathrm{~d}$. The models are valid for mixes with 0.35 to 0.42 water-to-binder ratio (W/B), $10 \%$ to $40 \%$ of pulverised fuel ash (PFA) as replacement of cement by mass, 0.02 to $0.06 \%$ of viscosity enhancer admixture (VEA), by mass of binder, and 0.3 to $1.2 \%$ of superplasticizer (SP), by mass of binder. The derived models that enable the identification of underlying primary factors and their interactions that influence the modelled responses of composite cement paste are presented. Such parameters can be useful to reduce the test protocol needed for proportioning of composite cement paste. This paper attempts also to demonstrate the usefulness of the models to better understand trade-offs between parameters and compare the responses obtained from the various test methods which are highlighted. The multi parametric optimization is used in order to establish isoresponses for a desirability function of cement composite paste. Results indicate that the replacement of cement by PFA is compromising the early compressive strength and up $26 \%$, the desirability function decreased.
\end{abstract}

Keywords: Compressive strength; Mini-slump; Plate cohesion meter; Pulverised fuel ash; Superplasticizer; Viscosity enhancer admixture; Water/Binder.

\section{INTRODUCTION}

Recently, there has been a growing interest in the use of cement paste containing VEA admixture for repairs, injection, embedding of anchors and post-tensioning, rock and oil-well grouting. A cement-based paste should be stable enough to reduce sedimentation, bleeding, and water dilution. Cement-based pastes are widely used in injection grouting of cracks in massive structures since their physical and mechanical properties can be easily controlled. This is assured by judicious choice of the type and fineness of cement, water-to-binder ratio, chemical and mineral admixtures [1]. The incorporation of one or more types of supplementary cement replacement materials (CRMs) such as pulverised fuel ash having different morphology and grain-size distribution values can improve particle size distribution and packing of solid particles, hence enhancing fluidity, stability and permeability. Improvements in the quality and uniformity of CRMs and the attention given to admixture formulations have greatly assisted progress.

Viscosity-enhancer admixtures (VEAs) are used to enhance the cohesion and stability of cement-based systems. Such VEAs are water-soluble polysaccharides that enhance the water retention capacity of paste [2,3]. Diutan gum is high molecular weight, microbial polysaccharides produced by aerobic fermentation and is different to welan gum. It is anionic polymers and its performance is influenced by the

*Address Correspondence to this Author at the School of Planning, Architecture and Civil Engineering, Queen's University Belfast, Belfast, Northern Ireland, UK; Tel: 442890 974013; Fax: 442890 663754; E-mail: m.sonebi@qub.ac.uk presence of superplasticizer (SP). Without the presence of SP, the biopolymer's anionic nature will cause them to have a tendency to adsorb out of the mix water onto the surface of cement [4] and with the presence of SP, it prevents this phenomena. Diutan gum had two-rhamnose side chain compare to welan gum which had only one rhamnose and one mannose. The molecular length of diutan gum is up to 3 times longer than welan gum, which the molecular weights of diu$\tan$ is about 2.88 to 5.18 million Daltons [5]. The use of VEA increases the yield value and plastic viscosity of the cement-based paste, thus necessitating an increase in water/binder or SP dosage to insure a low yield value necessary for proper penetrability and spreading $[6,7]$. The increase of W/B ratio results in decrease in mechanical properties, impermeability, and durability of pastes [6,7].

The use of PFA in cementitious paste represents a relatively mature technology, which has been utilised in a range of construction applications. Experience has shown that compared to neat Portland cement paste, PFA incorporation can effect improvements in rheology and provide the full range of engineering properties normally required $[6,8]$. Applications such as soil, rock, and oil-well grouting all require enormous amounts of cement and are therefore good examples of areas where some part of cement could be partially replaced by PFA to produce low cost, environmentally safe and durable pastes [8].

Several researchers have related the improvement in properties of cement-based paste to the enhancement in performance [1, 6-8]. For example, high-performance cement paste made with microfine cement with $0.6 \mathrm{~W} / \mathrm{B}$ and $2 \%$ of SP was used successfully for crack injection grouting of submerged structures $[1,9]$. 
The main objective of this study is to evaluate the effect of the water-to-binder ratio, the PFA content, the dosages of SP and VEA on the rheological properties and compressive strength at $3 \mathrm{~d}, 7 \mathrm{~d}$ and $28 \mathrm{~d}$ of composite pastes using statistical design approach and analysis of experiments $[10,11]$. The models can be used to evaluate the potential influence of adjusting mix variables on paste properties required to ensure successful development of composite paste. Such simulation can facilitate the test protocol needed to optimize composite paste with a given set of performance criteria that can be tried in the laboratory. Multiparametric optimization using the desirability function was used to establish response surfaces

\section{DEVELOPMENT OF FACTORIAL DESIGN AP- PROACH}

The technique of analysis used in this study was a $2^{\mathrm{k}-1}$ fractional experimental design was used to evaluate the influence of two different levels for each variable on the relevant paste properties [10]. Four key parameters that can have significant influence on mix characteristics of composite cement paste were selected to derive mathematical models for evaluating relevant properties $(\mathrm{k}=4$ in this study, thus the total of mixes for the fractional factorial was $2^{4-1}=8$ ). The four variables were W/B, PFA content, concentrations of SP and VEA. The modelled experimental region consisted of mixes ranging between coded variables of -1 to +1 (Table 1). The derived statistical models are valid for mixes made with ranges of W/B of 0.35 to $0.42,10 \%$ to $40 \%$ PFA, concentrations of VEA of 0.02 to $0.06 \%$, by mass of binder, and SP of 0.3 to $1.2 \%$, by mass of binder. The paste responses that were modelled were the mini-slump, the Marsh cone, the cohesion Lombardi meter, and the unit weight, and 3-d, 7-d and 28-d compressive strengths.

The eight mix combinations, expressed in coded values, considered in the experimental design composite pastes are listed in Table 2. Two-level factorial design does not allow an estimate of the experimental error unless some runs are repeated; thus four replicate central points were prepared to estimate the degree of experimental error for the modelled responses (Table 2 ). The central points consisted of mixes where variables were fixed in the middle level corresponding to: $25 \%$ PFA as replacement of cement, $0.8 \%$ SP (\% mass of binder), $0.04 \%$ VEA by mass of binder, and $0.39 \mathrm{~W} / \mathrm{B}$. The 18 mix combinations, expressed in coded and actual values, considered in the experimental design of pastes are listed in Table 2. The coded units of variables are calculated as follows.

$$
\text { Coded Factor }=\frac{(\text { Actual value }- \text { Factor means })}{\left[\frac{\text { Range of the factorial values }}{2}\right]}
$$

Coded $\mathrm{W} / \mathrm{B}=($ absolute $\mathrm{W} / \mathrm{B}-0.385) / 0.035$

Coded PFA $=($ absolute PFA -25$) / 15$

Coded SP $=($ absolute SP -0.75$) / 0.45$

Coded VEA $=($ absolute VEA -0.04$) / 0.02$

\section{MATERIALS AND MIX PROPORTIONS}

The paste mixes investigated in this study were prepared with Standard CEM I 42.5N Ordinary Portland cement (OPC) and PFA. OPC and PFA used conformed to BS
Table 1. Coded and Absolute Values for the Investigated Parameters

\begin{tabular}{|c|c|c|c|c|}
\hline Parameter & Range & $\mathbf{- 1}$ & $\begin{array}{c}\text { Central } \\
\text { Point }\end{array}$ & $\mathbf{+ 1}$ \\
\hline \hline W/B & 0.35 to 0.42 & 0.35 & 0.39 & 0.42 \\
\hline PFA (\%) & 10 to $40 \%$ & 10 & 25 & 40 \\
\hline VEA (\%) & 0.02 to $0.06 \%$ & 0.02 & 0.04 & 0.06 \\
\hline SP $(\%)$ & 0.3 to $1.2 \%$ & 0.3 & 0.8 & 1.2 \\
\hline
\end{tabular}

Table 2. Mix Proportion for Pastes used in the Two-Level Fractional Factorial Design

\begin{tabular}{|c|c|c|c|c|c|}
\hline & \multicolumn{5}{|c|}{ Coded values } \\
\hline & Mix & W/B & SP & PFA & VEA \\
\hline & & & $(\%)$ & $(\%)$ & $(\%)$ \\
\hline \multirow{8}{*}{ Levels of factors } & 1 & -1 & -1 & -1 & -1 \\
\hline & 2 & 1 & -1 & -1 & 1 \\
\hline & 3 & -1 & 1 & -1 & 1 \\
\hline & 4 & 1 & 1 & -1 & -1 \\
\hline & 5 & -1 & -1 & 1 & 1 \\
\hline & 6 & 1 & -1 & 1 & -1 \\
\hline & 7 & -1 & 1 & 1 & -1 \\
\hline & 8 & 1 & 1 & 1 & 1 \\
\hline \multirow{4}{*}{ Centre points } & 9 & 0 & 0 & 0 & 0 \\
\hline & 10 & 0 & 0 & 0 & 0 \\
\hline & 11 & 0 & 0 & 0 & 0 \\
\hline & 12 & 0 & 0 & 0 & 0 \\
\hline \multirow{6}{*}{$\begin{array}{c}\text { Points of verifica- } \\
\text { tion }\end{array}$} & 13 & 0.50 & 1.05 & 0.50 & -0.75 \\
\hline & 14 & 0.75 & 0.75 & 0.75 & -0.25 \\
\hline & 15 & 0.50 & 0.50 & 0.50 & -0.25 \\
\hline & 16 & 0.75 & 0.75 & 0.75 & -0.50 \\
\hline & 17 & -0.25 & 1.00 & 0.75 & 0.00 \\
\hline & 18 & -0.50 & 1.00 & 0.75 & 0.00 \\
\hline
\end{tabular}

EN197-1 and BS 3892, Part 1 and their chemical and physical properties are shown in Table 3 . The specific density of PFA was 2.1.

A superplasticizer produced on the basis of modified polycarboxylic ether Fluid Optima 100 from Chryso was used as aqueous solution with a solid content of $30 \%$ and specific gravity of 1.05 . Diutan gum was supplied by Kelcocrete which an anionic polysaccharide developed specifically for use with cementitious materials and is a natural high molecular weight gum produced by carefully controlled aerobic fermentation. 
Table 3. Chemical and Physical Properties of PFA and Cement

\begin{tabular}{|c|c|c|}
\hline & OPC & PFA Part 1 \\
\hline $\mathrm{SiO}_{2}(\%)$ & 20.8 & 50 \\
\hline $\mathrm{AL}_{2} \mathrm{O}_{3}(\%)$ & 5 & 30 \\
\hline $\mathrm{Fe}_{2} \mathrm{O}_{3}(\%)$ & 3.2 & 7 \\
\hline $\mathrm{CaO}(\%)$ & 63.7 & 2.3 \\
\hline $\mathrm{MgO}(\%)$ & 2.6 & 1.2 \\
\hline $\mathrm{K}_{2} \mathrm{O}(\%)$ & --- & 1.1 \\
\hline $\mathrm{Na}_{2} \mathrm{O}(\%)$ & --- & 0.08 \\
\hline $\mathrm{TiO}_{2}(\%)$ & --- & 1.1 \\
\hline $\mathrm{SO}_{3}(\%)$ & --- & 0.4 \\
\hline Chloride (\%) & --- & $<0.03$ \\
\hline Free $\mathrm{CaO}(\%)$ & 1.6 & --- \\
\hline LOI (\%) & 0.65 & 5.9 \\
\hline $\mathrm{Na}_{2} \mathrm{O}$ eq. $(\%)$ & 0.39 & --- \\
\hline Moisture content & --- & Nil \\
\hline Water requirement (\%) & --- & 94 \\
\hline Strength factor & --- & 0.85 \\
\hline Bulk Density & --- & $1.2-1.7 \mathrm{~g} / \mathrm{cm}^{2}$ \\
\hline Specific density & 3.15 & 2.1 \\
\hline $\begin{array}{l}\text { Compressive strength - } \\
\qquad 28 \mathrm{~d}(\mathrm{MPa})\end{array}$ & 62 & 60.7 \\
\hline Particle density $\left(\mathrm{kg} / \mathrm{m}^{3}\right)$ & 3150 & 2029 \\
\hline $\begin{array}{l}\text { Specific Surface area } \\
\qquad\left(\mathrm{m}^{2} / \mathrm{kg}\right)\end{array}$ & 385 & $5.8^{*}$ \\
\hline
\end{tabular}

*: \% retained on a $45 \mu$ m sieve; BS 3892 Part 1: 1997

\section{TESTING PROCEDURES}

All paste mixes were prepared in batches of $2 \mathrm{~L}$ that were mixed using a high-shear mixer having $4.5 \mathrm{~L}$ maximum capacity. The mixing water was $9.9 \pm 0.2^{\circ} \mathrm{C}$ to compensate for the heat generated during the mixing action. The pastes temperature following the end of initial mixing was at $14.2 \pm 0.3$ ${ }^{\circ} \mathrm{C}$. The cement and PFA were mixed with VEA. The mixing sequence consisted of adding all of the water and SP to the mixer. The binder mixed with VEA were then introduced gradually after 1 minute. The paste was mixed for 1 minute, followed by $30 \mathrm{~s}$ of rest and another mixing period of $3 \mathrm{~min}-$ utes.

Following the end of mixing, the temperature and specific gravity of the paste were measured, and the mini-slump, Marsh cone, Lombardi Plate, and the unit weight were determined. All tests were carried out between 5 and $15 \mathrm{~min}$ following the initial contact of binder with water and were done always in the same sequence. Three $50-\mathrm{mm}$ cubes were cast in laboratory environment $\left(\mathrm{T}=20 \pm 2^{\circ} \mathrm{C}\right)$ to determine the compressive strength at 3,7 and $28 \mathrm{~d}$.
The fluidity was evaluated using mini slump cone and Marsh cone having a 5-mm outlet diameter. The mini-slump cone has an upper diameter of $19 \mathrm{~mm}$, a lower diameter of $38.1 \mathrm{~mm}$, and height of $57.2 \mathrm{~mm}$ [12].

The Marsh cone test measures the flow time of a given volume of a paste through a cone of a standard size. The funnel Marsh cone used in this study has a capacity of 1200 $\mathrm{ml}$ and an internal orifice diameter of $5 \mathrm{~mm}$. The time needed for a paste sample to flow through the cone is proportional to the viscosity of the paste. The flow time increases with the increase in viscosity, and thereby, it becomes an index of fluidity. The flow time measurement is performed by taking a representative sample of $1100 \mathrm{ml}$ of paste, plugging the lower orifice of the cone, then pouring the sample through a fine sieve that can retain any clumps of cement particles. Once the paste is allowed to flow out of the funnel and into a graduated cylinder, the time of flow is recorded at $700 \mathrm{ml}$.

The cohesiveness of the slurry was measured with a Lombardi plate cohesion meter [13]. The apparatus consists of a thin steel plate $(100 \mathrm{~mm} \times 100 \mathrm{~mm} \times 1 \mathrm{~mm})$, on which the paste can stick, and an electronic scale. The clean dry plate was weighed and then submerged once into the slurry. The plate was then withdrawn and weighed again after any dropping of slurry stopped.

The unit weight of the paste was measured by a mud balance. This mud balance consists of a constant-volume sample cup with lid connected to a balance arm. A reader is moved along the balance arm to indicate the scale reading. There is a knife edge attached to the arm near the balance cup and a bubble level built into this knife edge for levelling the arm. It was possible to calculate the thickness of paste on each side of the Lombardi plate from the unit weight and the amount of paste sticking to the steel plate.

The compressive strength was determined on $50-\mathrm{mm}$ cubes. The specimens were demoulded one day after casting and were stored in water until testing at $3 \mathrm{~d}, 7 \mathrm{~d}$, and $28 \mathrm{~d}$.

\section{RESULTS AND DISCUSSION}

\section{Derived Statistical Models}

These results were analysed and plotted using an appropriate statistical software package [14]. The derived statistical models are summarized in Table $\mathbf{4}$ with correlation coefficients and probability (Prob.) $>|t|$ values. The estimates for each parameter refer to the coefficients of the model found by a least square approach. The Prob. $>|t|$ term is the probability of getting an even greater $t$ statistic, in absolute value, that tests whether the true parameter is zero. Probabilities less than 0.05 are often considered as significant evidence that the parameter is not zero, i.e. that the contribution of the proposed parameter has a highly significant influence on the measured response.

The presentation in Table 4 enables the comparison of various parameters. For the majority of the parameters, the probabilities that the derived coefficients of the various parameters influencing each response are limited to $5 \%$. This signifies that there is less than $5 \%$ chance or $95 \%$ confidence limit, that the contribution of a given parameter to the tested 
Table 4. Parameter Estimates of the Six Derived Models

\begin{tabular}{|c|c|c|c|c|c|c|}
\hline & \multicolumn{2}{|c|}{ Mini-Slump } & \multicolumn{2}{|c|}{ 1/SQ(Lombardi plate value)* } & \multicolumn{2}{|c|}{ Unit weight } \\
\hline & \multicolumn{2}{|c|}{$\mathrm{R}^{2}=0.95$} & \multicolumn{2}{|c|}{$R^{2}=0.98$} & \multicolumn{2}{|c|}{$\mathrm{R}^{2}=0.98$} \\
\hline & Estimate & Prob. $>|t|$ & Estimate & Prob. $>|t|$ & Estimate & Prob. $>|t|$ \\
\hline Intercept & 110 & & 1.76 & & 1844 & \\
\hline $\mathrm{W} / \mathrm{B}$ & 6.12 & 0.001 & 0.25 & 0.0002 & -42.8 & $<0.0001$ \\
\hline PFA & NS & -- & NS & -- & -66.3 & $<0.0001$ \\
\hline SP & 11.6 & $<0.0001$ & 0.41 & $<0.0001$ & NS & -- \\
\hline \multirow[t]{3}{*}{ VEA } & -7.4 & 0.0006 & -0.39 & $<0.0001$ & NS & \\
\hline & \multicolumn{2}{|c|}{ 3-day f'c } & \multicolumn{2}{|c|}{ 7-day f'c } & \multicolumn{2}{|c|}{ 28-day f'c } \\
\hline & \multicolumn{2}{|c|}{$\mathrm{R}^{2}=0.86$} & \multicolumn{2}{|c|}{$\mathrm{R}^{2}=0.96$} & \multicolumn{2}{|c|}{$\mathrm{R}^{2}=0.95$} \\
\hline Intercept & 23.8 & -- & 35.5 & -- & 55.0 & \\
\hline $\mathrm{W} / \mathrm{B}$ & -6.74 & 0.0005 & -6.30 & $<0.0001$ & -7.24 & 0.0002 \\
\hline PFA & -5.3 & 0.0023 & -8.58 & $<0.0001$ & -12.11 & $<0.0001$ \\
\hline SP & NS & -- & NS & -- & NS & -- \\
\hline VEA & NS & -- & NS & -- & NS & -- \\
\hline
\end{tabular}

*: 1/square root (Lombardi plate) NS: None significant".

response exceeds the value of the specified coefficient. A negative estimate signifies that an increase of the given parameter results in a reduction of the measured response. For example, an increase in SP increases the mini-slump, i.e. increases the fluidity. The models in Table 4 give an indication of the relative significance of various parameters on each response. The majority of the models had high correlation coefficients $\left(\mathrm{R}^{2}\right)$ which most of them were up to 0.95 . The most of flow times through the Marsh cone were over 25 min and only mixes 4,7 and 8 had flow time values of 6.7 min, 23.6, and $18.1 \mathrm{~min}$, respectively. Therefore, there was not enough data to run the analysis of the results of Marsh cone. It can be concluded that the Marsh cone with $5 \mathrm{~mm}$ orifice was not suitable to measure the flow time and may be bigger orifices ( 8 or $10 \mathrm{~mm}$ ) can be used.

The model (for Y - mini-slump, Lombardi plate cohesion meter, unit weight, 3-d, 7-d and 28-d f' ${ }_{c}$ responses) associated with the two-level fractional factorial design in this case of four independent variables was as follows:

$$
Y=a_{0}+a_{1} W / B+a_{2} P F A+a_{3} S P+a_{4} V E A+\varepsilon
$$

where: $a_{0}$ denotes the overall mean; coefficients $a_{n}$ represent model constants (contribution of independent variables on the response), and $\varepsilon$ is the random error term representing the effects of uncontrolled variables. The values of coefficients an are shown in Table 4.

The derived statistical models of slurry for mini-slump, cohesion meter, unit weight and compressive strength at $3 \mathrm{~d}$, $7 \mathrm{~d}$, and $28 \mathrm{~d}$ are given in Eqs. (S1-S6).

Mini - slump $(\mathrm{mm})=110.1+11.6 \cdot \mathrm{SP}-7.4 \cdot \mathrm{VEA}+6.1 \cdot \mathrm{W} / \mathrm{B}$

$$
\begin{aligned}
1 / \sqrt{\text { Plate cohesion }} & \left(\mathrm{mm}^{-0.5}\right)=1.76+0.41 \cdot S P \\
& -0.39 \cdot V E A+0.25 \cdot W / B
\end{aligned}
$$

Unitweight $\left(\mathrm{kg} / \mathrm{m}^{3}\right)=1844-42.8 \cdot \mathrm{W} / \mathrm{B}-66.3 \cdot \mathrm{PFA}$

$$
\begin{gathered}
f^{\prime}{ }_{c} 3 d(M P a)=23.8-6.7 \cdot W / B-5.3 \cdot P F A \\
f^{\prime}{ }_{c} 7 d(M P a)=35.5-8.6 \cdot P F A-6.3 \cdot W / B \\
f^{\prime}{ }_{c} 28 d(M P a)=55.0-12.1 \cdot P F A-7.2 \cdot W / B
\end{gathered}
$$

The analysis of the variance for the derived models is given in Table 5. The Fisher distribution has also been used to evaluate the significance of the derived model. The $\mathrm{F}$ value has compared to $F_{v 1, k, n-k-1}$ from the Fisher table where $v 1$ is the degree of freedom, $\mathrm{k}$ is the number of unknown coefficients in the model and $\mathrm{n}$ is the number of experiments. For a $95 \%$ confidence interval, $\mathrm{F}_{0.05,3,4}$ value is 6.59 . The $\mathrm{F}$ values for mini-slump and the Lombardi plate cohesion (Table 5) are greater than 6.59 (48.6 \& 102.4). Thus, the effects of SP, VEA and W/B are considered highly significant on the derived model responses. For unit weight, $\mathrm{f}_{\mathrm{c} 3 \mathrm{~d}}, \mathrm{f}_{\mathrm{c}}{ }_{\mathrm{7d}}$, and $\mathrm{f}_{\mathrm{c} 28 \mathrm{~d}}$, the Fisher values are greater than 6.94 (vs. 160.6, 25.4, 84.9 and 84). Therefore, the effects of W/B and PFA are considered highly significant on the derived model responses.

Table 6 indicates the average measured responses of the four replicate pastes, coefficients of variation (COV), as well as the estimated errors with $95 \%$ confidence limit for each of the measured properties. The estimated errors for the minislump, Lombardi cohesion meter, unit weight, 3-d f' ${ }_{c}, 7-\mathrm{d} \mathrm{f}_{\mathrm{c}}$ 
,28-d f' ${ }_{c}$ were $\pm 1.5 \mathrm{~mm}, \pm 0.002 \mathrm{~mm}, \pm 1.3 \mathrm{~kg} / \mathrm{m}^{3}, \pm 1.7$ $\mathrm{MPa}, \pm 1.7 \mathrm{MPa}$, and $\pm 4.1 \mathrm{MPa}$, respectively. The relative experimental errors for mini-slump, and unit weight are shown to be limited to 1.3 to $1.5 \%$ and $0.8 \mathrm{~mm}$ for Lombardi cohesion meter. On the other hand, the relative error for the 3-d f' ${ }_{c}, 7-d f^{\prime}{ }_{c}$ and 28-d f' ${ }_{c}$ was limited to 7\%.

Table 5. Analysis of Variance of Mini-Slump, Lombardi Plate, unit weight, 3-d, 7-d and 28-d f'

\begin{tabular}{|c|c|c|c|c|c|c|}
\hline & \multicolumn{3}{|c|}{ Mini-Slump } & \multicolumn{3}{|c|}{$1 / \sqrt{(\text { LombardiPlate })}$} \\
\hline & DF & SS & Fvalue & $\mathrm{DF}$ & SS & Fvalue \\
\hline Model & 3 & 1816.4 & 48.6 & 3 & 3.05 & 102.4 \\
\hline Error & 3 & 6.8 & & 3 & 0.0001 & \\
\hline \multirow[t]{2}{*}{ Total } & 11 & 2248.7 & & 11 & 3.38 & \\
\hline & \multicolumn{3}{|c|}{ Unit weight } & \multicolumn{3}{|c|}{$\mathbf{f}_{\mathbf{c} 3 \mathrm{~d}}$} \\
\hline Model & 2 & 49733 & 160.6 & 2 & 586.8 & 25.42 \\
\hline Error & 3 & 0.0001 & & 3 & 8.6 & \\
\hline \multirow[t]{2}{*}{ Total } & 11 & 51788 & & 11 & 679.5 & \\
\hline & \multicolumn{3}{|c|}{$f_{c 7 d}^{\prime}$} & \multicolumn{3}{|c|}{$f_{c 28 d}^{\prime}$} \\
\hline Model & 2 & 905.8 & 84.9 & 2 & 1592.8 & 84 \\
\hline Error & 3 & 8.5 & & 3 & 48.7 & \\
\hline Total & 11 & 980.2 & & 11 & 1685.8 & \\
\hline
\end{tabular}

DF: Degree of freedom, SS: Sum of Squares, F value: Fisher value

Table 6. Repeatability of Test Results

\begin{tabular}{|c|c|c|c|}
\hline & Mini-Slump & $\begin{array}{c}\text { Lombardi } \\
\text { Test }\end{array}$ & Unit Weight \\
\hline Mean $(\mathrm{N}=4)$ & $98.8 \mathrm{~mm}$ & $0.233 \mathrm{~mm}$ & 1862 \\
\hline $\operatorname{COV}(\%)$ & 1.5 & 0.8 & 0.2 \\
\hline \multirow[t]{2}{*}{ Est. error* } & $1.53 \mathrm{~mm}$ & $0.002 \mathrm{~mm}$ & 2.5 \\
\hline & 3-d f ${ }_{c}^{\prime}$ & 7-d $\mathbf{f}_{c}^{\prime}$ & 28-d f', \\
\hline Mean $(\mathrm{N}=4)$ & $24.2 \mathrm{MPa}$ & $38.9 \mathrm{MPa}$ & $57.5 \mathrm{MPa}$ \\
\hline $\operatorname{COV}(\%)$ & 7.0 & 4.3 & 7.0 \\
\hline Est. error* & $1.72 \mathrm{MPa}$ & $1.72 \mathrm{MPa}$ & $4.11 \mathrm{MPa}$ \\
\hline
\end{tabular}

\section{ACCURACY OF THE PROPOSED MODELS}

The accuracy of each of the proposed models was determined by comparing predicted-to-measured values obtained with mixes prepared with six selected randomly mixes and to verify the ability of the proposed models to predict responses (Table 2). All tests were carried out with the same materials and under the same test procedures used for the previous mixes. The predicted-to-measured ratio for mini-slump, Lombardi plate cohesion meter, unit weight, and compressive strength at 3,7 and $28 \mathrm{~d}$ are summarized in Table 7.
The ratio between predicted and various measured properties of paste ranges between 0.90 and 1.01 thus indicating good accuracy for the established models to predict the minislump, Lombardi plate cohesion meter, unit weight and compressive strength at $3 \mathrm{~d}, 7 \mathrm{~d}$ and $28 \mathrm{~d}$. In general, the proposed models for mini-slump, unit weight and the compressive strength appear to be satisfactory in predicting the fluidity and the strength with low scattering between the measured and predicted values. On the other hand, the average value of predicted/measured ratio of plate cohesion meter was slightly lower (0.90).

Table 7. Predicted-to-measured ratios of established models

\begin{tabular}{|c|c|c|c|c|c|c|}
\hline & $\begin{array}{c}\text { Mini- } \\
\text { Slump }\end{array}$ & $\begin{array}{c}\text { Lomb. } \\
\text { Plate }\end{array}$ & $\begin{array}{c}\text { Unit } \\
\text { Weight }\end{array}$ & 3-d f'c & 7-d f'c & $\begin{array}{c}\text { 28-d } \\
\text { f'c }^{\prime}\end{array}$ \\
\hline \hline $\begin{array}{c}\text { Verifi- } \\
\text { cation } \\
\text { points }\end{array}$ & 1.01 & 0.90 & 0.98 & 0.99 & 0.96 & 0.98 \\
\hline
\end{tabular}

\section{ISORESPONSES OF THE PROPOSED MODELS OF THE KEY VARIABLES}

\section{Mini-Slump}

The proposed statistical models can therefore be used to evaluate the effect of a group of variables on the properties affecting the quality of cement paste. This permitted the calculation of the isoresponse curves from the parameters under study over the experimental domain and the optimisation of their effects. As shown in Table 4, the mini-slump is influenced, in order of significance, by the dosage of SP, the dosage of VEA, and W/B. The dosage of SP is shown to exhibit the greatest effect on the mini-slump due to better dispersion of cement particles in the system. The increase in SP has approximately 1.9 and 1.6 times greater influence on increasing mini-slump than the increase in $\mathrm{W} / \mathrm{B}$, and the reduction in the dosage of VEA, respectively (11.6 vs. 6.1 and -7.4). For example, the effect of increasing $\mathrm{W} / \mathrm{B}$ ratio on minislump vs. the dosage of SP, when dosages of PFA were fixed at $40 \%$ and VEA fixed at $0.02 \%, 0.04 \%$ and $0.06 \%$, respectively, is shown in Fig. 1. For any given W/B ratio and dosages of PFA and VEA fixed at $40 \%$ and $0.02 \%$, respectively, the mini-slump increased significantly when the dosage of SP increased. Similarly, the mini-slump increased when W/B increased while the dosages of VEA and SP, and the proportion of PFA were fixed. On the other hand, the mini-slump reduced when the dosage of VEA increased while the dosage of SP, and W/B were kept constant (Fig. 1). This is due to the water retention and the entanglement and intertwining of VMA polymer chains. The effects of W/B, SP and VEA on composite paste corresponded with the findings of other researchers $[6,7,16]$.

\section{Lombardi Plate Cohesion Meter}

As shown in Table 4, Lombardi plate cohesion meter is influenced, in the order of significance, by the dosages of SP and VEA, and W/B. By comparing the effect of SP and VEA dosages on the plate cohesion meter, the increase of dosage of SP can then be interpreted to have approximately similar influence on the reduction of the Lombardi plate cohesion value than the increase in VEA (0.41 vs. -0.39), for given 


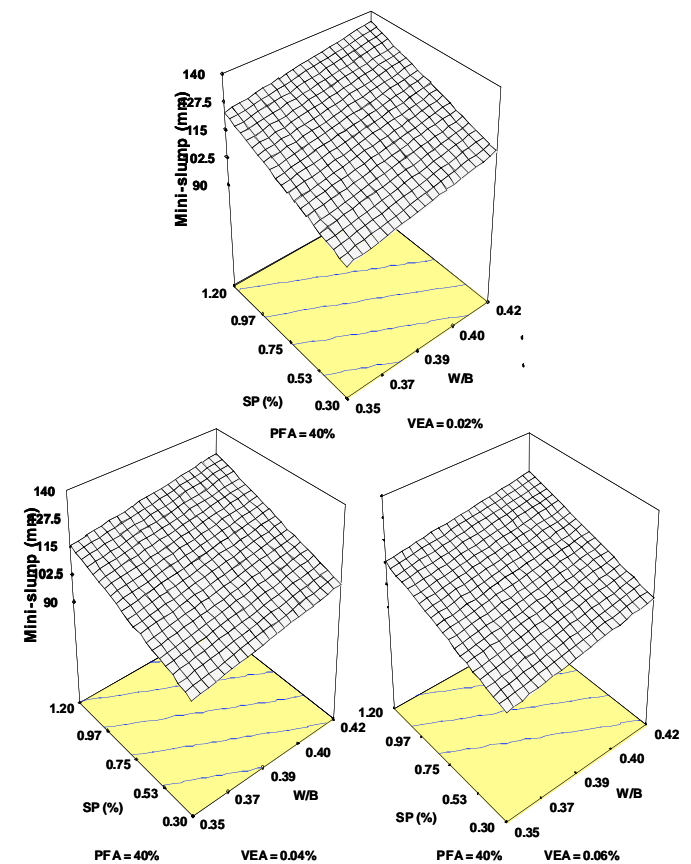

Fig. (1). Response surfaces of mini-slump test.

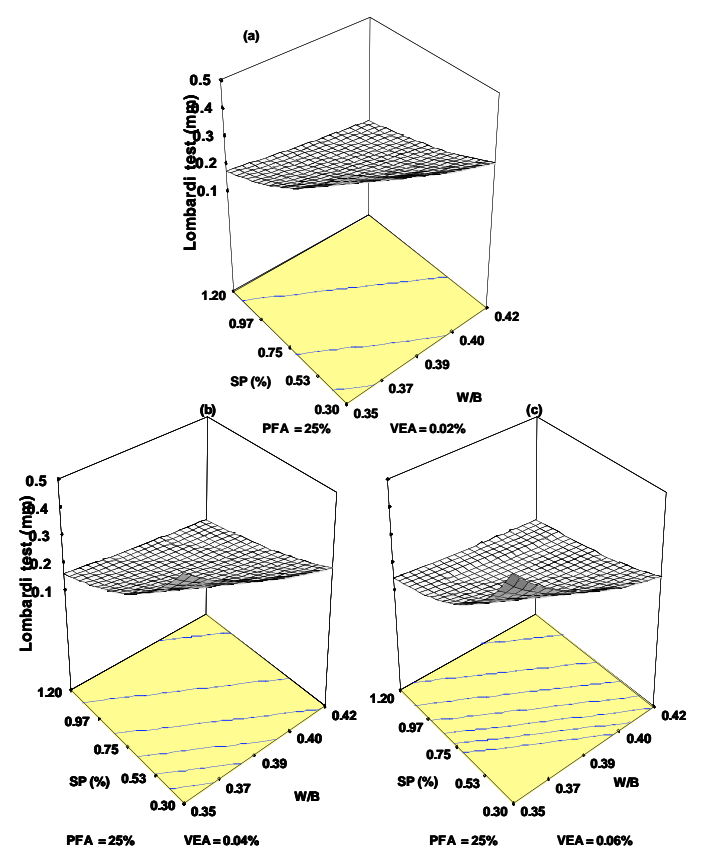

Fig. (2). Response surfaces of Lombardi plate cohesion meter.

that the W/B and the proportion of PFA are held constant. Fig. 2 shows an example of isopresponse curves of the Lombardi plate cohesion meter vs. W/B and SP and VEA dosages ranged from $0.02 \%$ to $0.06 \%$ and for fixed proportion of PFA of $25 \%$. It can be observed that for example for fixed VEA of $0.02 \%$, the increase of $\mathrm{W} / \mathrm{B}$ and SP led to a reduction of Lombardi plate cohesion (Fig. 2. (a)), and similarly the same effect for dosage of VEA at $0.04 \%$ and $0.06 \%$ (Figs. 2 (b) \& (c). This is due to the decrease of the cohesiveness of the grout having lower yield stress [7]. However, for any given $\mathrm{W} / \mathrm{B}$, and dosages of SP and PFA, the increase of the dosage of VEA exhibited an increase of Lombardi plate cohesion (Fig. 2) which is attributed to the entanglement and intertwining of VMA polymer chains and association of water between adjacent chains. The effects of $W / B$, SP and VEA confirm other findings on cement paste containing limestone powder [7]. This simple test could be used to give a good indication about the yield stress of cement paste.

\section{Unit Weight}

The two variables, W/B and the proportion of PFA (\%), were the only variables shown in the statistical models (Table 4) to have a significant effect on unit weight. As expected, the increase of $\mathrm{W} / \mathrm{B}$ and the proportion of PFA led to a reduction of unit weight which can be related to the increase of the porosity by increasing W/B and the lower specific density of PFA compare to cement (2.1 vs. 3.15).

\section{Compressive Strengths}

The ANOVAs given in Table 4 show that the effect of W/B is highly significant on compressive strength at 3, $7 \mathrm{~d}$ and $28 \mathrm{~d}$ and has the was just slightly more significant than the proportion of PFA on compressive strength at $3 d(-6.7$ vs. -5.3). The compressive strengths decreased, as W/B increased. The proportion of PFA was also highly significant on compressive strength showed a greater influence at 7 and $28 \mathrm{~d}$ (8.6 and 12.1 vs. 5.3 for $3 \mathrm{~d}$ ). The increase of the proportion of PFA led to a reduction in compressive strength at 3,7 and $28 \mathrm{~d}$. This is due to the pozzolanic reaction of PFA which occurs relatively slowly at normal temperature relative to Portland cement. The effect of the proportion of PFA on compressive strength at $28 \mathrm{~d}$ was more than 2 times comparing to compressive strength at $3 \mathrm{~d}(-12.1$ vs. -5.3$)$. It was reported that both of these variables reduced the compressive by other research [7, 14]. Figure 3 shows the isoresponse of the compressive strength at 7 and $28 \mathrm{~d}$ vs. W/B and the proportions of PFA. For example, for paste made with W/B of 0.39 and $25 \%$ PFA and given dosages of SP and VEA, the predicted compressive strengths at $7 \mathrm{~d}$, and $28 \mathrm{~d}$ were 36 $\mathrm{MPa}$, and $55 \mathrm{MPa}$, respectively.

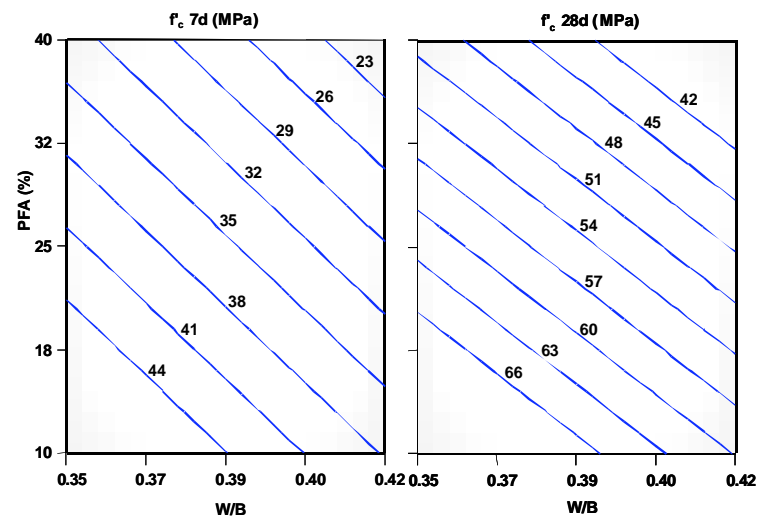

Fig. (3). Response surface of compressive strength at $3 d, 7 \mathrm{~d}$ and $28 \mathrm{~d}$.

\section{Trade-off between SP and VEA}

Contour responses showing the influence of SP and VEA dosages on mini-slump and Lombardi plate cohesion meter for pastes made with fixed W/B of 0.38 and $25 \%$ of PFA are 
presented in Fig. 4. As expected, for a given SP dosage, the contour diagrams of Fig. 4 indicate that the increase in VEA dosage reduces the mini-slump while the plate cohesion meter increases. For example, for mini-slump of $110 \mathrm{~mm}$, a mix paste with $0.62 \%$ of SP and $0.028 \%$ of VEA can ensure the fluidity of $110 \mathrm{~mm}$. The increase in VEA dosage to $0.041 \%$ resulted in a reduction of mini-slump to $105 \mathrm{~mm}$. However, by increasing the SP from $0.62 \%$ to $1.06 \%$, the mini-slump can be re-established to $110 \mathrm{~mm}$. For the same mix, with $0.62 \%$ of SP and $0.028 \%$ of VEA, the increase in VEA dosage to $0.041 \%$ increased the Lombardi plate cohesion from $0.3 \mathrm{~mm}$ to $0.4 \mathrm{~mm}$. The Lombardi plate cohesion meter can be re-established to $0.3 \mathrm{~mm}$ by increasing SP dosage to $0.87 \%$.

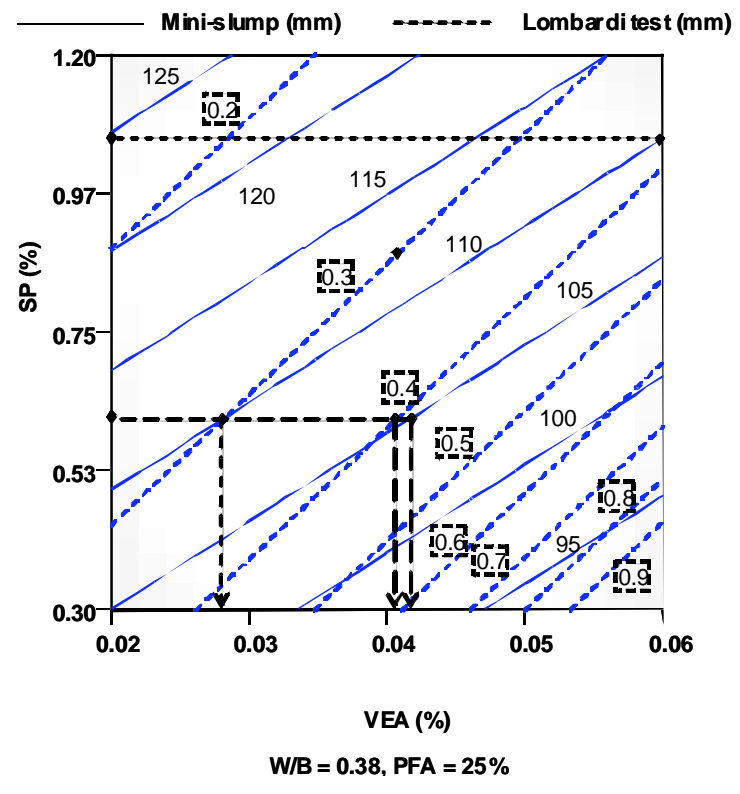

Fig. (4). Contour surfaces of mini-slump and Lombardi plate cohesion meter $(\mathrm{W} / \mathrm{B}=0.38, \mathrm{PFA}=25 \%)$.

\section{Correlation Between Mini-Slump and Plate Cohesion}

The flow of paste is very sensitive to its shear history. The above tests were carried out with extreme care in order to keep the shear history, the experimental procedures and their timing as constant as possible. Hence, it is interesting to assess the various possible correlation for example minislump and Lombardi plate cohesion.

Figure 5 shows the relationship between the mini-slump and Lombardi plate cohesion meter. The coefficient of correlation $\mathrm{R}^{2}$ was 0.65 and the relationship seemed to follow polynomial second-order model and shows that when the mini-slump increases the plate cohesion meter decrease. The relationship between mini-slump and the Lombardi plate cohesion meter was similar to some findings of other researchers testing cement paste incorporating limestone powder which they obtained better coefficient of correlation [7].

\section{Numerical Optimization for Desirability Function}

The numerical optimization can be made for a combination of factor levels that simultaneously satisfy the requirements placed on each of several responses. The simultaneous optimization each response have a low and high value as- signed to each goal. The goal field for responses is one five choices: none, maximum, minimum, target, or in a specified range. Factors are included in optimization, at their design range by default, or as a maximum, minimum of target goal. The goals are then combined into overall desirability function, which reflects the desirability ranges for each response. The desirable ranges are from zero to one for any given response which the numerical optimization using the software searches for the greatest overall desirability function. The value equal to one inside the experimental domain represents the ideal case and a zero indicates that one or more responses fall outside the desirable limits. Table 8 summarises the goals and weights for measured responses (mini-slump, Lombardi plate cohesion, compressive strength at $3 \mathrm{~d}, 7 \mathrm{~d}$, and $28 \mathrm{~d}$ ). To achieve these goals, Fig. 6 presents the response surfaces of the desirability function. It can be mentioned that the desirability function will change if the different goals have been selected. The response surface of Fig. 6 indicated that the desirability function decreased with the increase in the proportion of PFA and tended to increase when PFA was lower than $26 \%$. High W/B tended also to reduce the desirability function. The desirability function increased also when the W/B was limited to around 0.41 in order to maximize the compressive strength at $3 \mathrm{~d}, 7 \mathrm{~d}, 28 \mathrm{~d}$ and assuring in the same time high stability of paste as set the goals and weights (high fluidity and medium to high cohesion). It can be emphasis that the isoresponses of the desirability function will be changed if different goals or weights have been selected for the numerical optimization (different to those selected in Table $\mathbf{8}$, for example targeting values for min-slump and compressive strength at $3 \mathrm{~d}, 7 \mathrm{~d}$, and $28 \mathrm{~d}$ ).

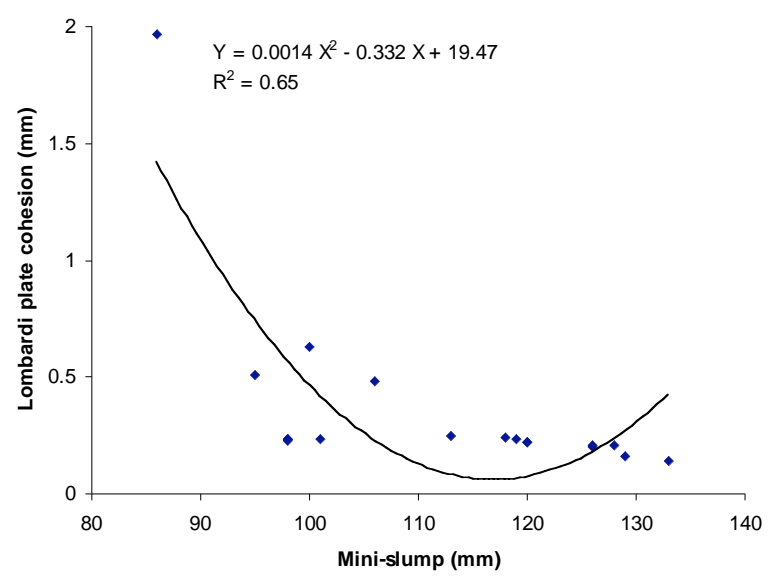

Fig. (5). Correlation between mini-slump and plate cohesion meter.

\section{CONCLUSIONS}

The effects of water/binder ratio, the proportion of PFA, and the concentrations of SP and VEA of composite pastes on the rheological properties and the compressive strength at $3 \mathrm{~d}, 7 \mathrm{~d}$ and $28 \mathrm{~d}$ were investigated. The proposed statistical models can simplify the test protocol required to optimize a given mix composite paste by reducing the number of trial batches needed to achieve a balance among mix variables. This is due to the use of the models in conducting simula- 
Table 8. Selected Criteria, Goals, and Weights Used in the Numerical Optimization

\begin{tabular}{|l|c|c|}
\hline Criteria & Goal & Weight \\
\hline W/B & $\begin{array}{c}\text { Within range from 0.35 } \\
\text { to } 0.42\end{array}$ & N.A. \\
\hline SP & Minimum & 1 \\
\hline PFA & $\begin{array}{c}\text { Within range from 10 } \\
\text { to 40 }\end{array}$ & N.A. \\
\hline VEA & Minimum & 1 \\
\hline Mini-slump & Maximum & 1 \\
\hline Lombardi plate & Maximum & 4 \\
\hline $\mathrm{f}^{\prime}$ at $3 \mathrm{~d}$ & Maximum & 5 \\
\hline $\mathrm{f}^{\prime}$ at $7 \mathrm{~d}$ & Maximum & 5 \\
\hline $\mathrm{f}^{\prime}{ }_{\mathrm{c}}$ at $28 \mathrm{~d}$ & Maximum & 5 \\
\hline
\end{tabular}

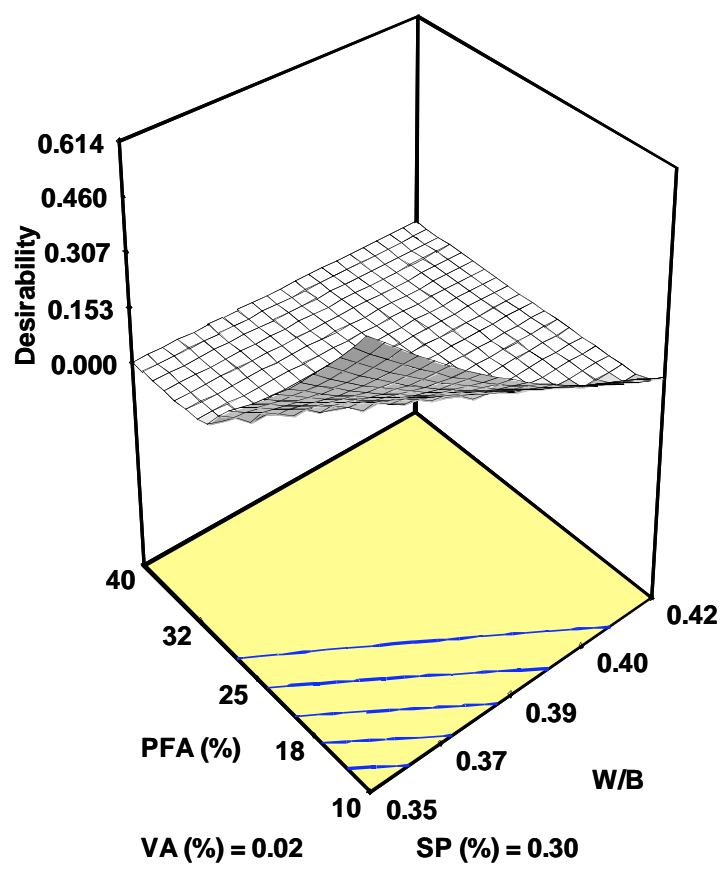

Fig. (6). Response surfaces of desirability function based on multiparametric numerical optimization for pastes incorporating PFA.

tions of various variables to secure adequate fluidity, cohesion and compressive strength. Based on the results of this paper, the conclusions can be drawn:

- The W/B ratio is shown to exhibit a great effect on mini-slump, Lombardi plate cohesion, and compressive strength at $3 \mathrm{~d}, 7 \mathrm{~d}$ and $28 \mathrm{~d}$. The increase in $\mathrm{W} / \mathrm{B}$ ratio let to an increase of fluidity, and a reduction in the Lombardi plate cohesion, unit weight and the compressive strength.

- The mini-slump, the Lombardi plate cohesion are dominated primarily by the dosage of SP. The increase in the dosage of SP led also to an increase in mini-slump and a reduction in the Lombardi plate cohesion which is due to the dispersion properties of SP. However, the dosage of SP had no effect on unit weight and compressive strength.
- The increase of dosage of VEA exhibited significant effect on both mini-slump and Lombardi plate cohesion. In fact, the increase in the dosage of VEA led to a reduction of mini-slump and an increase of the plate cohesion which is attributed to water retention and the entanglement and intertwining of VMA polymer chains.

- $\quad$ For a given W/B, and dosage of SP and VEA, the increase of the proportion of PFA let to reduction of compressive strength at $3 \mathrm{~d}, 7 \mathrm{~d}$, and $28 \mathrm{~d}$ which is due to the slowest pozzolanic reaction of PFA compared to cement. The PFA replacement of cement had a greater effect on compressive strength than the change of $\mathrm{W} / \mathrm{B}$ ratio particularly at $7 \mathrm{~d}$ and $28 \mathrm{~d}$.

- $\quad$ The desirability function decreased with the increase in the proportion of PFA and tended to increase when PFA was lower than $26 \%$. It is also reduced when W/B increased.

- $\quad$ The models established using a factorial design approach valid for pastes with 0.35 to $0.42 \mathrm{~W} / \mathrm{B}, 10 \%$ to $40 \%$ PFA, 0.02 to $0.06 \%$ VEA, and 0.3 to $1.2 \%$ SP. These models can also provide relationships between the forgoing results for mix optimization and quality control. The modelling and prediction of the response of others points in the experimental domain were therefore possible. Although the modelling are based on a given set materials, they can be easily used to generate other future results using other materials such as ground granulated blastfurnace slag, or silica fume, but the difference between the predicted and the measured values will than indicate the effect of the new materials on the accuracy of the proposed models. Additional research is needed to help the engineer to mix proportioning the cement composite paste using effectively and economically supplementary materials.

\section{ACKNOWLEDGEMENTS}

The authors would like to acknowledge the support and facilities provided by the School of Planning, Architecture and Civil Engineering at Queen's University Belfast to carry out this work. JWS Weis acknowledged for her contribution to report the experimental program.

\section{REFERENCES}

[1] P.E. James Warner . Practical handbook of grouting: soil, rock, \& structures. John Wiley \& Sons, Hoboken, New Jersey, 2004.

[2] T. Kawai . Non-dispersible underwater concrete using polymers, Marine Concrete, International Congress on Polymers in Concrete, Brighton, England, 1987 Chapter 11.5.

[3] V.A. Ghio, P.J.M. Monteiro, L.A. Demsetz .The rheology of fresh cement paste containing polysaccharide gums, Cement \& Concrete Research, vol. 24(2) pp. 243-249, 1994.

[4] A. Phyfferoen and C.B. Skaggs . Optimizing the use of welan gum in cementitious and gypsum-based systems. In ConChem Proceedings, Verlag für chemische Industrie, H. Ziolkowsky, Augsburg, 193-202, 1997.

[5] N. Sakata ,S. Yanai ,M. Yoshizaki ,A. Phyfferoen and A. Monty Evaluation of S-657 biopolymer as a new viscosity-modifying admixture for self-compacting concrete. Proceedings of the 2nd International Symposium on Self-Compacting Concrete, Ed. K. Ozawa, Ouchi, M., Tokyo, Japan, 229-236, 2001.

[6] M .Sonebi . Factorial design modelling of mix proportion parameters of underwater composite cement grouts. Cement Concrete Research, vol. 31(11),pp. 1553-1560, 2001.

[7] L. Svermova ,M. Sonebi and P.J.M. Bartos . Influence of mix proportions on rheology of cement grouts containing limestone powder, Cement, Concrete and Composites, vol. 25(7),pp. 737-749, 2003. 
[8] J.Mirza ,K. Saleh, V. Roy and M.S. Mirza . Use of high volume fly ash in grouting applications, Proceedings of the Third CANMET/ACI International Conference on High-Performance Concrete: Design and Materials and Recent Advances in Concrete Technology, SP-172 1997,pp. 281-298.

[9] K.H. Khayat and G. Ballivy . High-performance cement grout for underwater crack injection. Third CANMET/ACI International Conference on Performance of Concrete in Marine Environment, Ed. V.M. Malhotra, August, 1996, pp.138-162.

[10] D.C. Montgomery . Design and analysis of experiments, Sixth edition, John Wiley \& Sons, 2005.

[11] M. Sonebi ,P.J.M. Bartos, A. Tamimi . Application of factorial models to predict the effect of anti-washout admixture, superplasticizer and cement on slump, flow Time and washout resistance of underwater concrete, Materials and Structures, vol. 33 (229),pp. 317-323, 2000.
[12] D.L. Kantro . Influence of water reducing admixtures on properties of cement paste - A miniature slump test, Cement Concrete and Aggregates, CCAGDP, vol. 2 (2) pp. 95-102, 1980.

[13] G. Lombardi . The Role of cohesion in the cement grouting of rock. Proceedings of Fifteenth Congress on Large Dams. International Commission on Large Dams, Q.53, R.B., 3, Lausanne, 1985, pp. 235-261.

[14] Design-Expert 7.0 software for design of experiments. Stat-Ease Inc., Statistics Made Easy, USA, 2005.

[15] M. Sonebi . Medium strength self-compacting concrete containing fly ash: modelling using factorial experimental plans. Cement and Concrete Research, vol. 34(7),pp. 1199-1208, 2004.

[16] M. Nehdi ,S. Mindess, P. C. Aitcin . Statistical modelling of the microfiller effect on the rheology of composite cement pastes. $A d$ vances in Cement Research, vol. 9(33),pp. 37-46, 1997. 